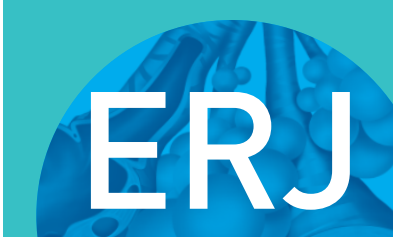

open research

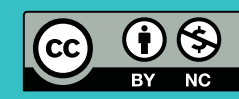

\title{
Technology for noninvasive mechanical ventilation: looking into the black box
}

\author{
Ramon Farré ${ }^{1,2,3}$, Daniel Navajas ${ }^{1,2,4}$ and Josep M. Montserrat ${ }^{2,3,5}$ \\ Affiliations: \\ ${ }^{1}$ Unitat Biofísica i Bioenginyeria, Facultat de Medicina, Universitat de Barcelona, Barcelona, Spain. \\ ${ }^{2}$ CIBER Enfermedades Respiratorias, Madrid, Spain. \\ ${ }^{3}$ Institut d'Investigacions Biomèdiques August Pi Sunyer, Barcelona, Spain. \\ ${ }^{4}$ Institut de Bioenginyeria de Catalunya, Barcelona, Spain. \\ ${ }^{5}$ Sleep Lab, Hospital Clínic, Universitat de Barcelona, Barcelona, Spain.
}

\section{Correspondence:}

Ramon Farré, Unitat Biofísica i Bioenginyeria, Facultat de Medicina, Casanova 143, 08036 Barcelona, Spain.

E-mail: rfarredub.edu

ABSTRACT Current devices for providing noninvasive respiratory support contain sensors and built-in intelligence for automatically modifying ventilation according to the patient's needs. These devices, including automatic continuous positive airway pressure devices and noninvasive ventilators, are technologically complex and offer a considerable number of different modes of ventilation and setting options, the details of which are sometimes difficult to capture by the user. Therefore, better predicting and interpreting the actual performance of these ventilation devices in clinical application requires understanding their functioning principles and assessing their performance under well controlled bench test conditions with simulated patients. This concise review presents an updated perspective of the theoretical basis of intelligent continuous positive airway pressure and noninvasive ventilation devices, and of the tools available for assessing how these devices respond under specific ventilation phenotypes in patients requiring breathing support.

@ERSpublications

Current devices for intelligent noninvasive ventilation should be tested to better understand clinical performance http://ow.ly/XAS6Z

Received: Jan 132016 | Accepted: Jan 172016

Conflict of interest: Disclosures can be found alongside the online version of this article at erj.ersjournals.com

Support statement: This work was partially supported by the Spanish Ministry of Economy and Competitiveness (Instituto de Salud Carlos III); grants PI14-00004, PI14/00416). Funding information for this article has been deposited with FundRef.

Copyright $\odot$ ERS 2016. This article is open access and distributed under the terms of the Creative Commons Attribution Non-Commercial Licence 4.0. 


\section{Introduction}

Devices aimed at noninvasively helping patients to breathe require very different levels of technological complexity depending on whether breathing support is provided to patients in the chronic or acute setting. The simplest application is for providing continuous positive airway pressure (CPAP) to avoid upper airway collapse in obstructive sleep apnoea. However, more complex devices are required for noninvasive mechanical ventilation (NIV) aimed at supporting the patient's inspiratory muscles, in patients with chronic respiratory diseases (e.g. neuromuscular, chest wall deformities, chronic obstructive pulmonary disease) [1] or with complex respiratory disorders during sleep (e.g. Cheyne-Stokes breathing, obesity hypoventilation syndrome) [2]. In most clinical applications these ventilatory support devices are prescribed to patients with chronic respiratory diseases and therefore are applied for very long periods, often for life. Hence, the treatment is not for application within the hospital environment but at the patient's home. This fact poses particular requirements on robustness and safety since these therapeutic devices are used with no (or limited) supervision by healthcare staff.

Noninvasive ventilatory devices developed during the early years of this new therapy were technologically simple, and therefore easy to evaluate and control, since they provided only very basic functions. Later on, the availability of miniaturised and cheap sensors and microprocessors made it possible to introduce a considerable amount of intelligence into noninvasive ventilatory devices [3-5]. This technological advancement was clinically relevant because it offered a new generation of machines, providing new variants of ventilation modes designed to increase patient comfort and hence improvement in adherence to therapy and clinical effectiveness. It goes without saying, however, that increasing the complexity of respiratory support devices also raised the need for both enhanced engineering control, in order to achieve reliable device performance, and clinical research, in order to assess the suitable adaptation of new breathing support modes to individual patients.

\section{The black box}

It is interesting to note that the technological complexity in noninvasive ventilatory devices has evolved quite differently to that in other respiratory medical devices. For instance, when introducing new flow sensor technologies and microprocessors in spirometers, engineers and manufacturers must comply with clear and widely accepted standards on the definition of indices and their measurement accuracy [6]. This constraint puts limits on the degrees of freedom in innovation but gives assurance to costumers on the expected performance of new devices. In contrast with the relatively well-regulated example of spirometry, manufacturers of noninvasive ventilatory support devices have almost unrestricted freedom to develop products with new and sophisticated features because: 1) legal regulations (e.g. CE mark or US Food and Drug Administration approval) for these devices only focus on a very few basic issues (e.g. pressure/ volume ranges, alarms) and 2) there are no widely accepted clinical statements or guidelines establishing a technical definition for acceptable ventilation modes and their corresponding quality assessment rules.

In this context, manufacturers develop devices incorporating distinctive and proprietary algorithms for new modes of ventilation with adaptive facilities for each patient based on variants and combinations of the classical pressure and volume control modes. Thus, different commercially available devices are claimed to be particularly suitable for providing more comfortable and clinically effective ventilatory support (e.g. by specific inspiratory ramps, cycle triggering, expiratory facilitation, leak correction) and/or for adapting in real time the ventilatory support to the patient's needs (e.g. detection of sleep apnoea events or changes in minute ventilation). Offering such a wide range of options on the market provides an excellent opportunity for choosing a device that could better fit a given patient. Nevertheless, the fact that manufacturers have no restrictions on the definitions of respiratory support modes is somewhat confusing for the end user. Indeed, in many cases, an algorithm or ventilation mode proposed by a manufacturer as being exclusive for its device is based on almost the same concept, but with a different registered trademark name, than the option proposed by another manufacturer [7]. The fact that 174 unique names for modes of ventilation were reported in 2010 [5], with new ones being added since then, illustrates how complex it is to distinguish ventilation devices, not only for clinicians but also for experts in this technology. The main problem from the user viewpoint (either physician, health professional or patient) is that most device algorithms are undisclosed since they play a central role in the commercial competition among manufacturers. Ignoring the details of how a device works does not allow the user to ascertain what the potential differences are between devices and whether these differences are reasonably substantial from a clinical viewpoint. In fact, for the conventional user, most current ventilatory support devices appear to be a black box $[7,8]$.

The need to evaluate and compare different devices is not exclusive to NIV technology. In general, assessment of medical devices or interventions can be carried out at three levels [5]: 1) a theoretical analysis of the principles and details of the device/procedure; 2) a bench test analysis to determine the 
actual device performance when subjected to well-known simulated patients; and 3) testing the usefulness of a device in the clinical arena with real patients presenting a wide range of pathological phenotypes. However, as a result of biological variability the results from clinical trials usually do not allow us to clearly disentangle the technical reasons explaining actual device performance in patients.

\section{Principles of devices for intelligent ventilatory support}

Analysing the principles of device function is of crucial importance in order to establish potential clinical usefulness [5]. Nevertheless, this analysis usually provides limited conclusions about actual device performance, particularly in the many cases that the detailed device algorithms remain undisclosed. Conceptually sound principles of functioning are a necessary but not a sufficient condition for good performance. Indeed, the actual quality of a device depends on the specific implementation of its theoretical basis, which is illustrated in figures 1 and 2, depicting examples of the rationale of intelligent CPAP or NIV devices, respectively.

\section{Automatic CPAP devices}

Automatic CPAP devices include built-in sensors and a microprocessor with algorithms to analyse the ventilation pattern of the patient in real time [3]. The aims of the device algorithm are: 1) to detect events among normal breathing cycles; 2) to distinguish whether a detected event is an artefact (e.g. cough, swallowing, mouth breathing) or a true pathologic breathing event (e.g. apnoea, hypopnoea, snoring, flow limitation); 3) to assess air leaks; and 4) to decide whether the value of CPAP applied to the patient should be maintained, increased or decreased (figure 1). In that way, the intelligent device is able to continuously tailor the nasal pressure applied to the patient's needs throughout the whole night. This general rationale is common for all automatic CPAP devices but it is not useful to evaluate how each device works unless the specific parameter definitions and thresholds in the different algorithm sections are known. For instance, how are events/artefacts detected, identified and classified; how many events of each type (and for how long) are required to modify the CPAP values applied to the patient; and how big is each change in pressure, and for how long is it applied [3]. Obviously, the actual performance of a given automatic CPAP device will depend on the specific implementation of its intelligence (also here the devil is in the details).

\section{Noninvasive mechanical ventilators}

Noninvasive ventilators are technologically more complex than CPAP devices since, in addition to applying different pressures in inspiration and expiration, they must correctly detect the patient's breathing effort activity to synchronically trigger these two phases of the ventilator cycle. The algorithms in NIV devices are also complex, since they should control the amount of inspiratory support according to patterns that depend on the ventilation modality and its specific settings [3,4]. As indicated by the general diagram in figure $2 \mathrm{a}$, an intelligent NIV device applies a support that depends on the settings in its control panel, on the mechanical properties of the patient's respiratory system and on his/her inspiratory activity.

Conceptually, all NIV modes are based on applying a given amount of positive pressure at the patient's airway opening (Pao). In case of negative pressure ventilators (not considered here), the situation is similar, with the ventilator applying a negative pressure at the chest wall surface, in the same direction as the inspiratory muscles. In its most simple formulation, the basic equation of motion governing breathing states that the respiratory system mechanics consists of a resistance ( $R$; mainly from the airways) and a

FIGURE 1 Schematic diagram depicting the function principles of a device for applying automatic continuous positive airway pressure (CPAP) (see text for explanation). $V^{\prime}$ and $P a o$ are flow and pressure measured at the patient's airway opening.

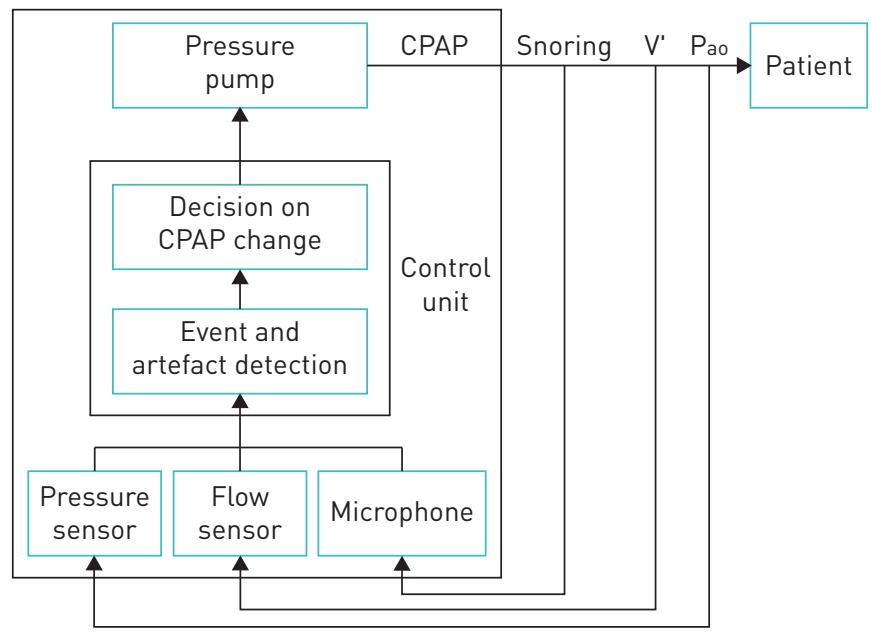


a)

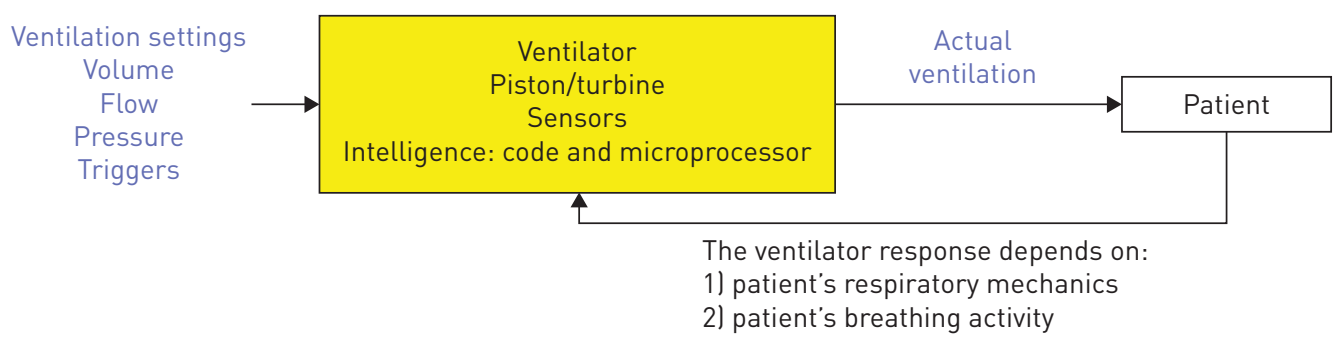

b)

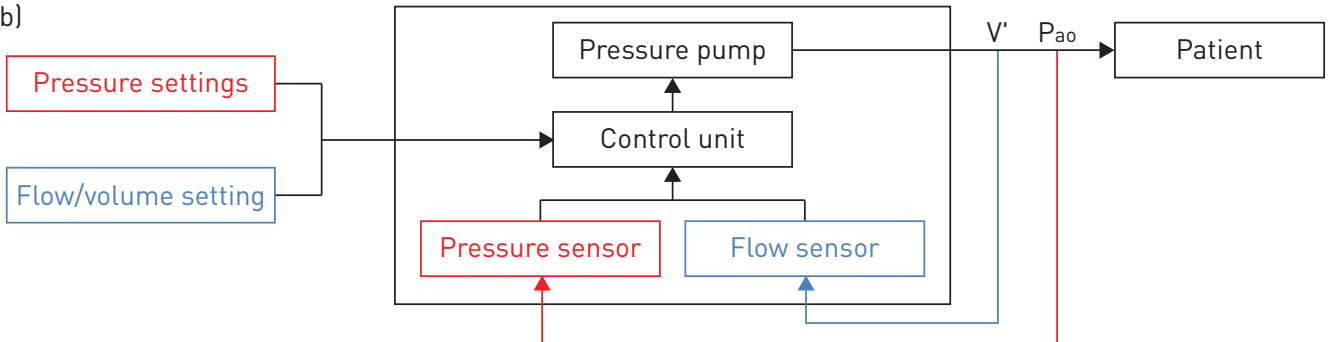

c)

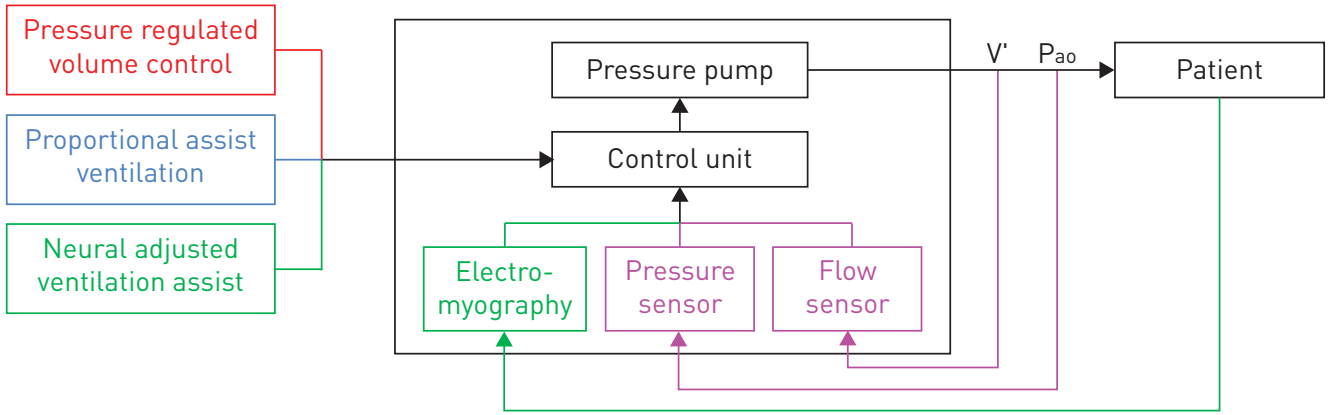

FIGURE 2 a) Schematic diagram of the function principles of a noninvasive mechanical ventilator. b) Diagram of conventional noninvasive mechanical ventilators. Pressure-controlled ventilators are described by the black and red components. Flow/volume-controlled ventilators are described by the black and blue components. $V^{\prime}$ and $\mathrm{Pao}$ are flow and pressure measured at the patient's airway opening. c) Diagram of advanced ventilators. Pressure regulated volume control ventilators are described by the black, red and magenta components. Proportional assist ventilation devices are described by the black, blue and magenta components. Neural adjusted ventilation assist devices are described by the black and green components. See text for explanations.

compliance (C; mainly from tissues) and that lung volume increase in inspiration is caused by application of a pressure $(\mathrm{P})$ across the system: $\mathrm{P}=\mathrm{R} \cdot \mathrm{V}^{\prime}+\mathrm{V} / \mathrm{C}$, where $\mathrm{V}^{\prime}$ and $\mathrm{V}$ are flow and volume, respectively [9]. During normal breathing this pressure $\mathrm{P}$ is the one generated by the inspiratory muscles (Pmus) exclusively: $\mathrm{P}=\mathrm{Pmus}$. During positive pressure noninvasive ventilation, respiratory muscles are supported by the ventilator, which is an external pump applying a positive inspiratory pressure at the airway opening, and therefore $\mathrm{P}=\mathrm{Pao}+\mathrm{Pmus}$ [9]. Positive pressure support is usually applied by a pump consisting on a servocontrolled (real-time feedback) turbine [10]. It is interesting to note that the fact that the ventilator is a pressure pump does not mean that NIV cannot be controlled by means of other variables (e.g. volume or inspiratory effort) [4].

As illustrated by figure $2 \mathrm{~b}$, the core of a noninvasive ventilator consists of three main sections: sensors, pressure pump and control unit. The most common ventilator sensors measure pressure at the airway opening and flow (and hence inspiratory volume by flow integration). The pressure pump is driven by the output signal from the control unit. The control stage is the more complex section and the one that allows the implementation of different modes of ventilation. For instance, in case of setting a pressure-controlled mode, the control unit continually compares what is the actual pressure measured at the airway opening and the target pressure and then drives the pressure source to achieve the desired value (figure $2 \mathrm{~b}$; black and red colours). In a flow/volume-controlled mode the control unit compares the measured inspiratory flow/volume and the target value, and then drives the pressure generator to achieve them (figure $2 \mathrm{~b}$; black and blue colours). 
Figure $2 c$ illustrates modes of ventilation more advanced than the pressure- and volume-controlled classical ones: pressure regulated volume control (PRVC), proportional assist ventilation (PAV) and neurally adjusted ventilatory assist (NAVA) [4]. PRCV is a pressure-controlled ventilation mode that ensures a given level of tidal volume or minute ventilation set in the control panel of the ventilator. To this end, the inspiratory pressure applied at the airway opening is not necessarily the same in each breathing cycle. Specifically, the control unit of the ventilator determines what level of inspiratory pressure is required to ensure the target ventilation [4]. Hence in PRCV the information captured by both the pressure and flow sensors is used to drive the pressure pump (figure 2c; black, magenta and red colours). This mode has the simplicity and comfort of pressure-controlled ventilation, with the added advantage of ensuring target ventilation is achieved, regardless of any changes either in the mechanical properties of the respiratory system of the patient, or in his/her neuromuscular drive.

Instead of controlling pressure or flow/volume, PAV aims to apply an open airway pressure that parallels the inspiratory pressure generated by the patient's muscles. Accordingly, it is expected that the machine works in a friendly way, since the patient will perceive the machine as an exact multiplier of his/her inspiratory muscles activity. To this end, airway pressure applied by PAV has two components, one proportional to flow and one proportional to volume, mimicking the resistive and elastic components of the respiratory driving equation: $P_{a o}=k_{1} \cdot V^{\prime}+k_{2} \cdot V, k_{1}$ and $k_{2}$ being setting constants (figure $2 \mathrm{c}$; black, blue and magenta colours) [11]. NAVA has the same aims than PAV (to provide a support reproducing the patient's effort), but with a different technical implementation [12]. The NAVA pressure applied at the airway opening is not determined from the measured pressure and flow signals but from the electromyographic recording of the patient's inspiratory muscles: $\mathrm{Pao}=\mathrm{k} \cdot \mathrm{Emd}$, where $\mathrm{k}$ is a constant and Emd is the integrated electromyography signal from the patient's diaphragm (figure $2 \mathrm{c}$; black and green colours). As in both PAV and NAVA, the ventilator support is directly dependent on the patient's inspiratory muscle action [13]; this mode is not suitable for all types of patient requiring NIV. Indeed, in patients with depressed respiratory drive or weak inspiratory muscles, these modes would result in potentially dangerous underventilation.

\section{Bench testing intelligent devices for ventilatory support}

Bench testing consists of assessing the actual performance of a device under well-defined conditions. In fact, this step is always necessary before launching a device on the market and it is a procedure that with more or less detail should be carried out by manufacturers. Bench testing is also carried out externally by agencies or users wanting to check that the device is working in accordance with the manufacturer specifications. In cases where the technical requirements of the device are perfectly standardised (as in the spirometry example mentioned), bench testing is used to confirm that the practical implementation of the design algorithms by the device hardware and software works properly. For instance, the typical bench test for spirometers is based on a servocontrolled syringe able to precisely reproduce a collection of representative spirometry curves covering the wide range of phenotypes found in clinical practice [6]. The spirometer under test, also a black box from the testing procedure viewpoint, is connected to the syringe and the index values measured by the device are compared with the known reference values reproduced by the simulated patients. This procedure allows both the assessment of the performance of a given spirometer and a comparison of different commercially available devices when subjected to exactly the same input. This sort of bench testing is relatively easy to carry out since the expected performance of the device is standardised. However, when the concept of bench testing is translated into the field of devices for noninvasive ventilatory support, the situation is not so clear since there are no standardised patient simulations nor definitions of what indices to measure and their error tolerance.

\section{Automatic CPAP devices}

Given that there are no widely accepted rules for testing automatic CPAP devices, no commercially available simulators of patients with sleep breathing disorders are available. Besides early passive patient models, current bench testing of automatic CPAP devices are carried out by simulated patients reproducing the different events found in sleep disordered breathing. Briefly, the simulated patient consists of a pump able to reproduce ventilation patterns and of an upper airway model able to reproduce the collapse events found in patients in a controlled and reproducible way. Figure 3a shows an example of settings used for bench testing automatic CPAP devices [14-16]. Realistic flow patterns previously recorded during polysomnography in obstructive sleep apnoea patients are reproduced by a computer-controlled pump. A servocontrolled valve, operating in synchrony with the flow generator, is able to simulate airway obstruction by reproducing resistance patterns previously recorded in patients experiencing apnoeas, hypopnoeas and flow limitation. A loudspeaker allows reproduction of snoring at the desired times during the breathing events. Moreover, in order to assess the performance of the automatic CPAP device in detecting typical artefacts such as mouth expiration, a second breathing circuit, in parallel to the main 


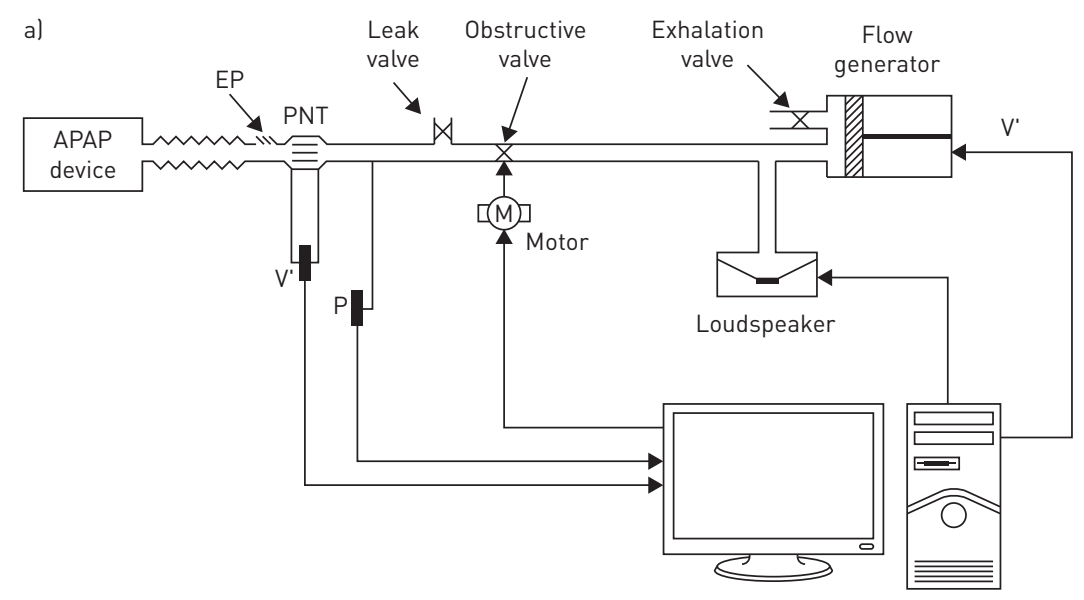

b)

Total test duration: 04:15 h

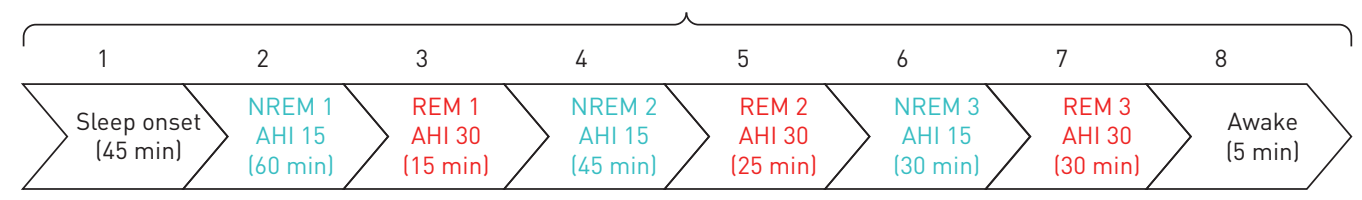
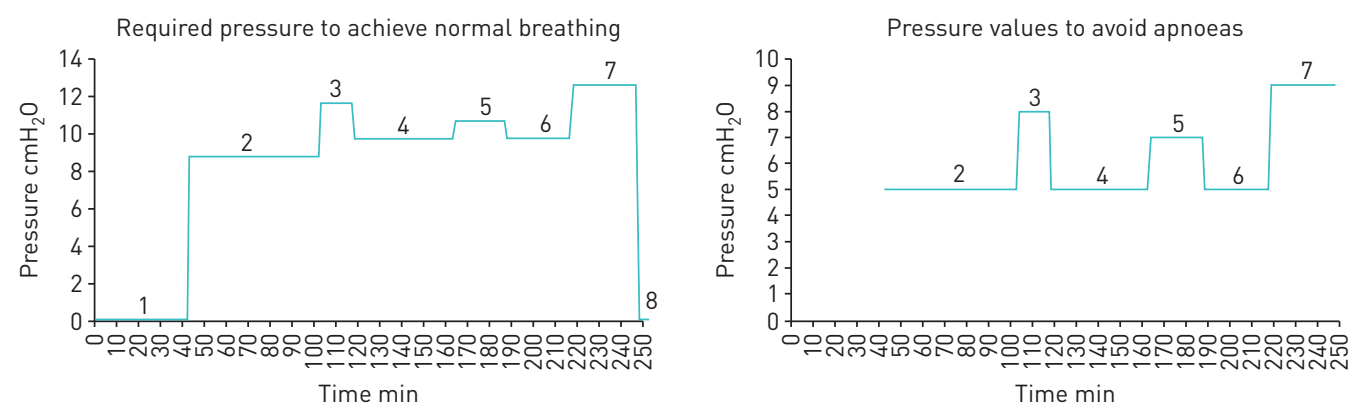

FIGURE 3 Bench test setting for automatic continuous positive airway pressure (APAP) devices. al System to simulate the breathing pattern and airway obstructions in patients with sleep disordered breathing. $V^{\prime}$ : flow; P: pressure; EP: exhalation port; PNT: pneumotachograph. Reproduced from [15] with permission from the publisher. b) Diagram of the different sleep sections in an example of whole-night simulated patient (see text for explanation).

circuit, allows simulation of mouth breathing. An additional exhalation valve in this second breathing circuit is electronically controlled and synchronised with the flow generator and the obstructive valve. During mouth expiration, the exhalation valve is opened, allowing the expired air to flow through the simulated mouth route while the obstructive valve is closed. A leak valve is used for simulating leaks through the mask usually found during conventional CPAP treatment in patients.

This computer-controlled simulated patient is active since it is able to respond to the CPAP applied at the airway opening. Indeed, depending on the pressure measured by the transducer (figure $3 \mathrm{a}$ ), the events simulated change. A specific patient can be defined to exhibit different events for different ranges of nasal pressures. For instance: apnoeas (for CPAP $<5 \mathrm{cmH}_{2} \mathrm{O}$ ), severe hypopnoeas (CPAP $\geqslant 5$ and $<7 \mathrm{cmH}_{2} \mathrm{O}$ ), mild hypopnoeas (CPAP $\geqslant 7$ and $<9 \mathrm{cmH}_{2} \mathrm{O}$ ), flow limitation (CPAP $\geqslant 9$ and $<12 \mathrm{cmH}_{2} \mathrm{O}$ ) and normal breathing ( $\mathrm{CPAP} \geqslant 12 \mathrm{cmH}_{2} \mathrm{O}$ ). It is interesting to note that active upper airway mechanics can also be simulated by means of analogue collapsible segments representing a Starling resistor [17]. A patient simulation improvement based on the setting in figure 3a has been recently proposed [18]. It consists of simulating an entire night composed of different realistic sleep stages, each one characterised by different collapsible properties of the upper airway [19]. As shown in the example in figure 3b, each sleep stage is characterised by different upper airway collapsibility (apnoea-hypopnoea index, pressure to avoid apnoeas and pressure to normalise breathing). This improved model allows simulation of almost any kind of patient, making it possible to test automatic CPAP devices when subjected to different sleep disordered breathing phenotypes from specific subpopulations (e.g. in obesity, females and the elderly) [20-26]. 
As expected from the absence of guidelines and/or regulations and the associated freedom of manufacturers to define the details of the algorithms to respond to sleep breathing events, considerably different results are observed when commercially available automatic CPAP devices are subjected to bench testing. Figure 4 shows examples of the response of currently available automatic CPAP devices when subjected to a whole-night patient model (such as the one in figure 3b). The pressure changes of each device throughout the whole test varied considerably in the magnitude of pressure response to obstructive events, the time taken to increase pressure during disrupted breathing, and device behaviour during the initial simulated wake period (grey lines in figure 4). Whereas device A did not normalise breathing in the simulated patient (pressure below the blue line) and at time around $100 \mathrm{~s}$ did not avoid apnoeas (pressure below the red line), devices $\mathrm{B}$ and $\mathrm{C}$ were able to fully normalise breathing. However, device B applied a pressure considerably higher than required, and device $\mathrm{C}$, which was able to adjust the applied pressure closely above the minimum value to normalise breathing, unexpectedly increased pressure during the initial awake period. It is interesting to note that the bench test literature reports differences among devices regardless of the model used to simulate upper airway collapsibility (computer-controlled valve $[16,18]$ versus Starling resistor [26, 27]). Differences between device performance are not necessarily a sign of dysfunction but the natural result of having different built-in algorithms and thresholds in each device. The fact that from the user viewpoint these devices appear as "black boxes" makes bench testing particularly interesting for the understanding of how a given automatic CPAP device responds under a well-defined set of events, thereby helping to predict whether it would be well suited for an individual patient's phenotype.

\section{Noninvasive mechanical ventilators}

Tools and expertise for bench testing of noninvasive ventilators are more common than for automatic CPAP devices. First, the most obvious reason explaining this fact is that technologies for NIV were developed earlier. Secondly, and more importantly, although NIV is not for life support, it is applied to patients with a certain degree of chronic respiratory failure and therefore quality control for NIV must be particularly effective [29-31]. This has led to an extensive body of literature reporting results on the bench testing of NIV devices. Until recent years, most of these tests were carried out with experimental settings assembled by researchers since realistic patient simulators were unavailable on the market. Bench testing NIV requires a rather complex patient simulator: 1) it should present realistic and tunable resistive and elastic properties so that it can be ventilated with physiological flow/volumes when applying conventional ventilation pressures, and 2) the simulated patient must be active, in other words it must generate inspiratory pressures mimicking the breathing activity of patients supported by NIV. An example of a bench test setting for NIV is shown in figure $5 \mathrm{a}$, where the authors combined a resistance-compliance passive model with a customised pressure pump to mimic inspiratory efforts [32]. However, the most recent NIV bench tests have been carried out by computerised active patient simulators that are now available on the market (figure 5b) [33-36].

Whereas conventional passive patient models to be ventilated are built with analogue resistors and air containers with compliant walls, advanced patient simulators are based on a computer-driven piston pump. The piston position, and hence the corresponding lung volume change, is driven by a motor unit according to the pressure applied at entrance of the system (corresponding to the airway opening) and the values of patient resistance and compliance set into the system. When the ventilator under test is connected to the simulated patient (at the entrance of the piston pump), the following actions are carried out in real time by the patient simulator: 1) a transducer continuously measures the pressure $\mathrm{P}$ applied by the ventilator; 2) the computer solves the equation of movement $\left(\mathrm{P}=\mathrm{R} \cdot \mathrm{V}^{\prime}+\mathrm{V} / \mathrm{C}\right)$ to calculate the values of ventilator flow $\mathrm{V}^{\prime}$ and volume $\mathrm{V}$ induced by the actual pressure $\mathrm{P}$ on a resistance-compliance system with the simulated values set in the control panel; and 3) the computer moves the piston position accordingly.
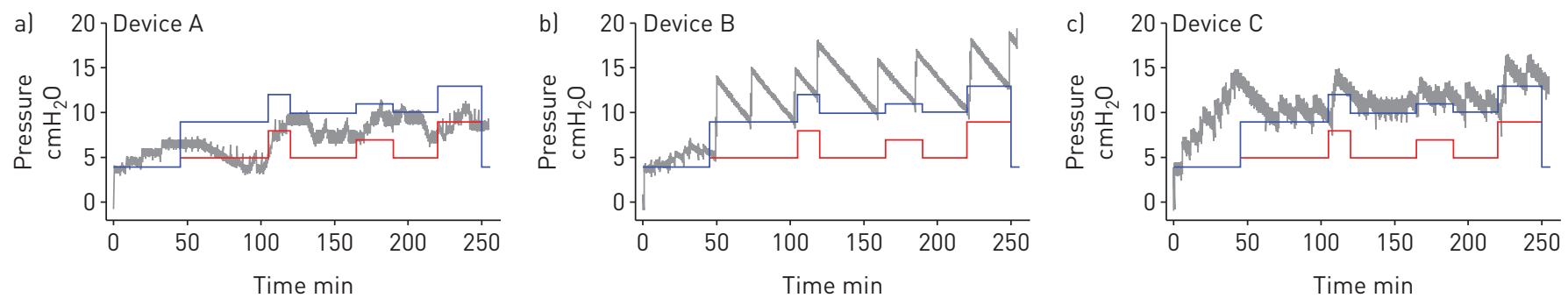

FIGURE 4 Pressure applied by different automatic continuous positive airway pressure devices in a bench test over a full simulated night lgrey

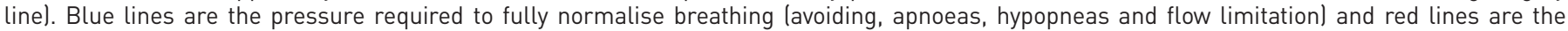
pressure required to avoid apnoeas. A device that delivered pressures above the blue line achieves full breathing normalisation, while if it delivered pressures just above the red line only obstructive apnoeas were overcome. 
a)

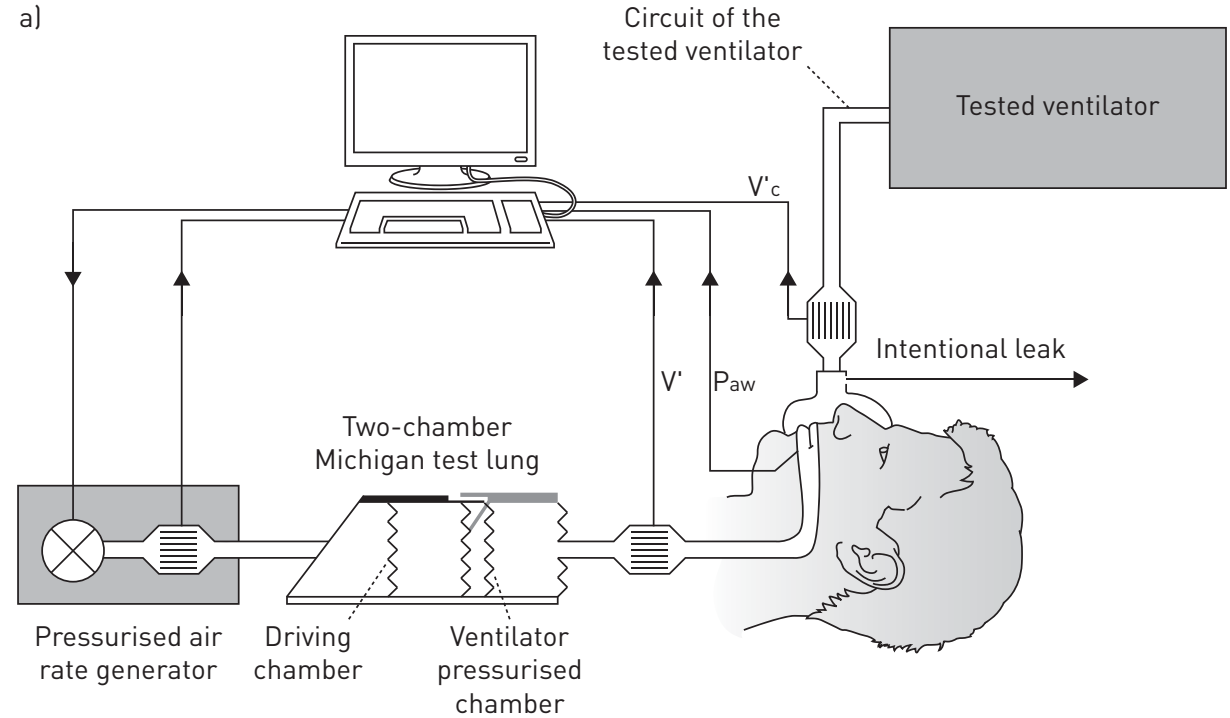

b)

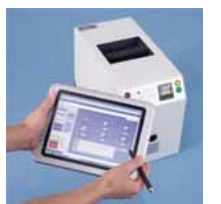

ASL 5000 Breathing
Simulator

IngMar Medical

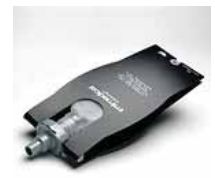

EasyLung

imtmedical

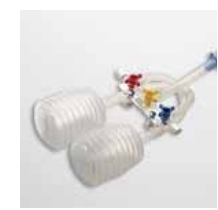

InfantLung

imtmedical

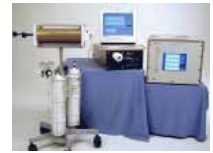

Lung Simulators

Hans Rudolph Inc

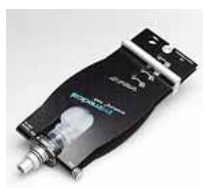

SmartLung Adult

imtmedical

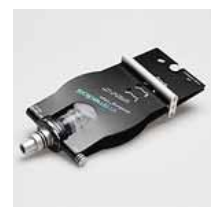

SmartLung Infant

imtmedical

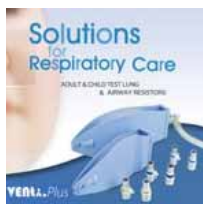

Venti.Plus Test Lung

and Airway Resistors

A Plus Medical

FIGURE 5 al Experimental system to carry out a bench test of noninvasive ventilators. V': flow into the lung chamber; V'c: flow delivered by the ventilator; Paw: airway pressure. Reproduced from [36] with permission from the publisher. b) Commercially available lung simulators to test mechanical ventilators (www. ersbuyersguide.org/features/category/lung-simulation). Some patient simulators for ventilation are passive (resistance-compliance systems) and some of them are computerised patient models allowing to easily simulate lung resistance and compliance and to activate inspiratory pressures simulating the patient's muscles activity.

From the viewpoint of the mechanical ventilator under test, this simulator has a response that is indistinguishable from the one of an analogue system with physical resistance and compliance. As compared with any conventional analogue model, such a computer-controlled patient simulator gives the user almost unlimited freedom to set values of resistance and compliance ranging within the whole possible spectrum in clinical practice (children versus adult and healthy versus obstructive/restrictive patients). Even more importantly, this type of patient simulator may perfectly mimic not only a simple resistance-compliance system, but more realistic and complex respiratory mechanical models including serial/parallel inhomogeneous lungs with or without viscoelastic tissue components. To this end, the basic equation of movement to be solved $\left(\mathrm{P}=\mathrm{R} \cdot \mathrm{V}^{\prime}+\mathrm{V} / \mathrm{C}\right)$ should be simply replaced by the equation of the more 

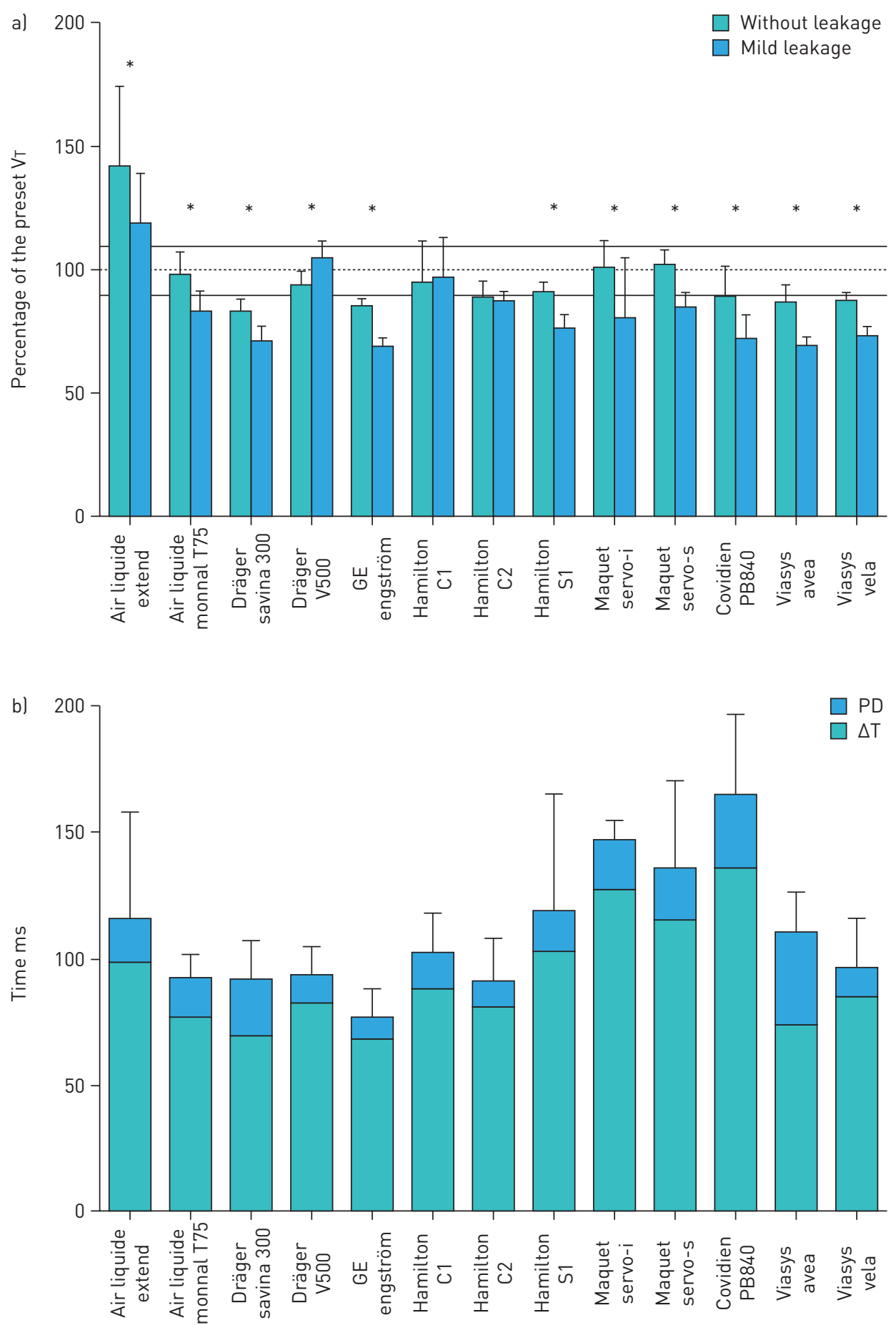

FIGURE 6 Results from a recent bench test of 13 noninvasive ventilators using a computer-controlled active patient simulator. a) Proportion of the preset tidal volumes $(\mathrm{V} T)$ actually delivered, without leakage and with mild continuous leakage. *: $p<0.05$ between conditions without leakage and with mild continuous leakage. b) Trigger performance in noninvasive pressure support ventilation. Inspiratory delays, triggering delay $(\Delta T)$ and pressurisation delay (PD), in response to moderate and strong inspiratory efforts, without leakage and with calibrated, mild and severe continuous leakage. Reproduced from reference [36] with permission.

realistic mechanical model to be simulated. Another key feature of computer-controlled patient simulators is that including the inspiratory pressure induced by the patient's muscles is extremely simple. In the case that, as indicated above, the pressure value used to solve the equation of movement is simply the airway opening pressure imposed by the ventilator connected to the simulated patient $(\mathrm{P}=\mathrm{Pao})$, it is obvious that the simulation would correspond to a passive patient (no breathing activity). To simulate the more 
complex condition of a patient subjected to ventilatory support, the simulator user can define any pattern of inspiratory muscle pressure (Pmus) and then the pressure value used to solve the equation of motion, and hence drive the piston, is $\mathrm{P}=\mathrm{Pao}+\mathrm{Pmus}$. Once again, computer-controlled patient simulators are more flexible than most analogue pumps used to superimpose breathing activity. In fact, any kind of muscle effort can be defined digitally, including specific inspiratory activity (e.g. breathing cycles, irregular respiration, deep inspirations/sights), artefacts (e.g. cough, speaking) and even expiratory efforts.

As NIV is very complex, both because the technical details of ventilators and the variety of patient phenotypes, a considerable number of important aspects need to be investigated when assessing the performance of noninvasive ventilatory devices $[33,36,37]$. One central question is whether the ventilator is able to provide the target pressure/volume under different practical conditions. Although the ventilators are servocontrolled and theoretically must apply the demanded pressure/volume, target achievement can be compromised in strenuous conditions $[38,39]$, namely: 1) when patients present very high/low values of resistance/compliance, respectively, 2) when caused by the almost always unavoidable air leaks at the noninvasive interface (usually a mask) [40], or 3) when the inspiratory/expiratory pressure induced by the patient muscles is particularly intense. Figure 6a shows the results of a recent bench test [36] indicating that the percentage of target volume actually provided to the patient by current ventilators depends on the device (ranging from $70 \%$ to $140 \%$ of the target tidal volume when tested with or without mild leakage). A crucial feature of NIV that can decrease patient tolerance and clinical effectiveness, and may even result in treatment intolerance and rejection, is triggering. Indeed, whereas providing a target pressure/ventilation magnitude that is slightly different from the prescribed value can be more or less tolerable, cases in which ventilator triggers inspiration/expiration asynchronically with the patient effort can present a major problem. Adequacy of triggering critically depends on the ventilator algorithm to detect the breathing patient activity and to decide the exact time for triggering each ventilator cycle from the information actually collected in real time (measured pressure and flow/volume). Triggering is a delicate process considerably affected by leaks and by the irregular inspiratory activity of the patient (e.g. deep inspirations, pauses and expiratory efforts). Accordingly, bench testing the triggering performance of noninvasive ventilators is of particular importance [41]. Figure 6b shows that in current NIV modes, both inspiratory delay and mean pressurisation delay in triggering considerably differed among ventilators (ranging 78-165 ms and 9-37 ms, respectively) [36]. Given that the ideal ventilator, i.e. the one providing perfect performance of all indices under any condition, does not exist, bench testing is a helpful tool to provide insight into whether a specific device/mode could be most suitable for a particular respiratory pathology or could better adapt to the specific needs of an individual patient.

\section{Conclusions}

Current devices for providing noninvasive respiratory support (for both sleep breathing disturbances and for chronic respiratory failure) are technically complex. Bench tests are a useful tool for better understanding the details of how the operating principles of a device have been implemented, and how the device actually performs when subjected to well-defined simulated patients. Bench testing is an important preliminary/complementary step in looking into the black box of current devices for noninvasive ventilatory support. This technical analysis can provide very deep insight and clues for the interpretation of device performance. However, final assessment of noninvasive ventilatory devices in real patients is the definitive touchstone.

\section{References}

1 Hannan LM, Dominelli GS, Chen YW, et al. Systematic review of non-invasive positive pressure ventilation for chronic respiratory failure. Respir Med 2014; 108: 229-243.

2 Elliott MW. Non-invasive ventilation during sleep: time to define new tools in the systematic evaluation of the technique. Thorax 2011; 66: 82-84.

3 Farre R, Montserrat JM. Principles of CPAP and auto-adjusting CPAP devices. Breathe 2008; 5: 43-50.

4 Chatburn RL, Mireles-Cabodevila E. Closed-loop control of mechanical ventilation: description and classification of targeting schemes. Respir Care 2011; 56: 85-98.

5 Mireles-Cabodevila E, Hatipoglu U, Chatburn RL. A rational framework for selecting modes of ventilation. Respir Care 2013; 58: 348-366.

Miller MR, Hankinson J, Brusasco V, et al. Standardisation of spirometry. Eur Respir J 2005; 26: 319-338.

Rabec C, Langevin B, Rodenstein D, et al. Ventilatory modes. What's in a name? Respir Care 2012; 57: 2138-2139. Brown LK. Autotitrating CPAP: how shall we judge safety and efficacy of a "black box"? Chest 2006; 130: 312-314. Farré R, Escarrabill J, Montserrat JM. Noninvasive ventilation treatment of sleep related breathing disorders. In: Kushida CA, ed. Handbook of Sleep Disorders. 2nd Edn. New York, Informa Healthcare, 2009; pp. 399-408.

10 Thille AW, Lyazidi A, Richard JC, et al. A bench study of intensive-care-unit ventilators: new versus old and turbine based versus compressed gas-based ventilators. Intensive Care Med 2009; 35: 1368-1376.

11 Younes M. Porportional assist ventilation, a new approach to ventilator support. Theory. Am Rev Respir Dis 1992 145: 114-120.

12 Navalesi P, Longhini F. Neurally adjusted ventilatory assist. Curr Opin Crit Care 2015; 21: 58-64. 
13 Kacmarek RM. Proportional assist ventilation and neurally adjusted ventilatory assist. Respir Care 2011; 56: 140-148.

14 Farré R, Montserrat JM, Rigau J, et al. Response of automatic continuous positive airway pressure devices to different sleep breathing patterns: a bench study. Am J Respir Crit Care Med 2002; 166: 469-473.

15 Rigau J, Montserrat JM, Wöhrle H, et al. Bench model to simulate upper airway obstruction for analyzing automatic continuous positive airway pressure devices. Chest 2006; 130: 350-361.

16 Isetta V, Navajas D, Montserrat JM, et al. Comparative assessment of several automatic CPAP devices' responses: a bench test study. ERJ Open Res 2015; 1: 00031-2015.

17 Abdenbi F, Chambille B, Escourrou P. Bench testing of auto-adjusting positive airway pressure devices. Eur Respir J 2004; 24: 649-658.

18 Isetta V, Montserrat JM, Santano R, et al. Novel approach to simulate sleep apnoea patients for evaluating positive pressure therapy devices. PLOS One 2016; in press [DOI: 10.1371/journal.pone.0151530].

19 Penzel T, Möller M, Becker HF, et al. Effect of sleep position and sleep stage on the collapsibility of the upper airways in patients with sleep apnea. Sleep 2001; 24: 90-95.

20 Valencia-Flores M, Bliwise D, Guilleminault C, et al. Gender differences in sleep architecture in sleep apnoea syndrome. J Sleep Res 1992; 1: 51-53.

21 Ware JC, McBrayer RH, Scott JA. Influence of sex and age on duration and frequency of sleep apnea events. Sleep 2000; 23: 165-170.

22 O'Connor C, Thornley KS, Hanly PJ. Gender differences in the polysomnographic features of obstructive sleep apnea. Am J Respir Crit Care Med 2000; 161: 1465-1472.

23 Series F, Marc I. Importance of sleep stage- and body position-dependence of sleep apnoea in determining benefits to auto-CPAP therapy. Eur Respir J 2001; 18: 170-175.

24 Woehrle H, Graml A, Weinreich G. Age- and gender-dependent adherence with continuous positive airway pressure therapy. Sleep Med 2011; 12: 1034-1036.

25 Russell T, Duntley S. Sleep Disordered breathing in the elderly. Am J Med 2011; 124: 1123-1126.

26 Tan H-L, Gozal D, Kheirandish-Gozal L. Obstructive sleep apnea in children: a critical update. Nat Sci Sleep 2013; 5: 109-123.

27 Zhu K, Roisman G, Aouf S, et al. All APAPs are not equivalent for the treatment of sleep disordered breathing: a bench evaluation of eleven commercially available devices. J Clin Sleep Med 2015; 11: 725-734.

28 Zhu K, Aouf S, Roisman G, et al. Pressure-relief features of fixed and autotitrating continuous positive airway pressure may impair their efficacy: evaluation with a respiratory bench model. J Clin Sleep Med 2015; in press [PMID: 26564383].

29 Farré R, Giro E, Casolive V, et al. Quality control of mechanical ventilation at the patient's home. Intensive Care Med 2003; 29: 484-486.

30 Farre R, Lloyd-Owen SJ, Ambrosino N, et al. Quality control of equipment in home mechanical ventilation: a European survey. Eur Respir J 2005; 26: 86-94.

31 Farre R, Navajas D, Prats E, et al. Performance of mechanical ventilators at the patient's home: a multicentre quality control study. Thorax 2006; 61: 400-404.

32 Louis $\mathrm{B}$, Leroux $\mathrm{K}$, Isabey $\mathrm{D}$, et al. Effect of manufacturer-inserted mask leaks on ventilator performance. Eur Respir J 2010; 35: 627-636.

33 Carteaux G, Lyazidi A, Cordoba-Izquierdo A, et al. Patient-ventilator asynchrony during noninvasive ventilation: a bench and clinical study. Chest 2012; 142: 367-376.

34 Olivieri C, Costa R, Conti G, et al. Bench studies evaluating devices for non-invasive ventilation: critical analysis and future perspectives. Intensive Care Med 2012; 38: 160-167.

35 Luján M, Sogo A, Pomares X, et al. Effect of leak and breathing pattern on the accuracy of tidal volume estimation by commercial home ventilators: a bench study. Respir Care 2013; 58: 770-777.

36 Garnier M, Quesnel C, Fulgencio J-P, et al. Multifaceted bench comparative evaluation of latest intensive care unit ventilators. British Journal of Anaesthesia 2015; 115: 89-98.

37 Zhu K, Kharboutly H, Ma J, et al. Bench test evaluation of adaptive servoventilation devices for sleep apnea treatment. J Clin Sleep Med 2013; 9: 861-871.

38 Fauroux B, Leroux K, Pépin JL, et al. Are home ventilators able to guarantee a minimal tidal volume? Intensive Care Med 2010; 36: 1008-1014.

39 Oscroft NS, Smith IE. A bench test to confirm the core features of volume-assured non-invasive ventilation. Respirology 2010; 15: 361-364.

40 Borel JC, Sabil A, Janssens JP, et al. Intentional leaks in industrial masks have a significant impact on efficacy of bilevel noninvasive ventilation: a bench test study. Chest 2009; 135: 669-677.

41 Conti G, Gregoretti C, Spinazzola G, et al. Influence of different interfaces on synchrony during pressure support ventilation in a pediatric setting: a bench study. Respir Care 2015; 60: 498-507. 